\title{
Accommodation and pleasantness of different forms of transcutaneous electrical nerve stimulation in individuals with nonspecific lumbar pain
}

\begin{abstract}
Objective: To evaluate the time, number of accommodation and the pleasantness for different forms of application of transcutaneous electrical nerve electrical stimulation (TENS) in nonspecific low back pain. Methods: A quantitative, randomized, crossover and comparative study was performed. Data were collected over a period of three weeks with a sample of twenty individuals with nonspecific low back pain who underwent three electrostimulation sessions with the Conventional TENS, Burst and VIF, held once per week. The individuals of the research were instructed to report the first and subsequent accommodation, and were subsequently questioned on the form they deemed most enjoyable.
\end{abstract}

Results: In relation to the first accommodation, it was verified that there was a significant difference $(\mathrm{p}<0.05)$ when compared to the Conventional and Burst groups. Regarding the number of accommodation, a significant statistical difference $(\mathrm{p}<0.05)$ was observed when comparing the Conventional and VIF groups. There was no statistically significant difference in the pleasantness.

Conclusion: The form of TENS VIF accommodated less, but the time required for accommodation was not higher than the others. There was nothing more preferred form of stimulation of volunteers.

Keywords: Transcutaneous electric nerve stimulation, physical therapy modalities, perception, sensory thresholds.
Volume 4 Issue 3 - 2019

\section{Elisangela Reway Costa, Fernanda Roth, Gabrieli Pauli,Valmir Barbosa Gozzblr, Maria das Graças Anguera, Gladson Ricardo Flor Bertolini \\ laboratory of the Research and Study Group of injuries and} physiotherapeutic Resources, State university of western paraná, Brazil

\section{Correspondence: Gladson Ricardo Flor Bertolini,} laboratory of the Research and Study Group of injuries and physiotherapeutic Resources, State university of Western paraná (unioeste) Cascavel, pR, Brazil,

Email gladson_ricardo@yahoo.com.br

Received: January II, 2019 | Published: May 10, 2019

\section{Introduction}

Nonspecific low back pain is considered to be among of the major health problems in industrialized countries. ${ }^{1}$ This type of low back pain is characterised by the absence of structural alteration, ie, there is no reduction of disc space, nerve root compression, bone or joint injury, scoliosis or pronounced lordosis that can lead to pain in the spine. They are characteristic of nonspecific low back pain: pain in weight, worsening with physical exertion mainly in the afternoon, relieved with the rest, absence of neurological alterations and muscular contracture and antalgic posture, associated with inactivity and inadequate posture..$^{2-4}$

Despite controversies, use of physiotherapeutic resources is widely used for the treatment of non-specific low back pain, ${ }^{5}$ and for transcutaneous electrical nerve stimulation (TENS) is acclaimed positive results in pain and disability for this population. ${ }^{6}$ Such a form of stimulation is a non-invasive treatment primarily utilized to promote analgesia. It involves the transmission of electrical energy from an external stimulator to the peripheral nervous system through surface electrodes connected to the skin. ${ }^{7,8}$ Acting on the peripheral mechanoceptors, the stimulus being carried by the large diameter $\mathrm{A} \beta$ fibers, to the set of interneurons, which acts to inhibit the retransmission of the painful stimuli carried by the fibers $\mathrm{A} \delta$ and $\mathrm{C}$, both of narrow diameters, closing the gates of the pain, ${ }^{9}$ but the mechanisms of endogenous opioid-mediated pain control and central modulation, $7,8,10$ serotonin release, aspartate and glutamate reduction on the medulla are also considered mechanisms. ${ }^{11}$ The TENS is applied in a variety of frequencies, intensities and pulse duration, being the most used are: high frequency (Conventional), low frequency (acupuncture), Burst and brief intense. ${ }^{12,13}$

About TENS, one of the subjects considered in the literature is the process of neural accommodation that occurs when a physiological response is continuously diminished by the repetitive application of the same stimulus. As an initial response, receptors act with high pulse frequency, whereas when the stimulus becomes constant, the pulse frequency decreases progressively. Maintaining the frequency of the stimulus is fundamental for the appearance of accommodation. With respect to the pleasantness it is known that the subjects feel different frequencies of therapeutic currents of diverse forms. Therefore, the subjective sensation of each individual must be taken into account when choosing the frequency, one should seek the best balance between the result and the minimum of discomfort for each individual. ${ }^{14,15}$

Although TENS is widely discussed in the literature, with evaluations of different parameters, on pain and other symptoms, ${ }^{8,10,16,17}$ few studies address variations in the process of accommodation and pleasantness for this modality, ${ }^{18,19}$ thus generating a lack of consensus on the behavior of different frequencies in relation to such variables. In this context, the objective of the present study was to evaluate the time, number of accommodation and the pleasantness for different forms of TENS application for individuals with nonspecific low back pain.

\section{Materials and methods}

The present study is characterized as quantitative, randomized, 
cross-sectional and comparative among the different forms of TENS electrostimulation application. Data collection was performed at the Physiotherapy Rehabilitation Clinic (CRF) of the Universidade Estadual do Oeste do Paraná - UNIOESTE - in the city of Cascavel, Paraná, Brazil, in a period of four weeks, the sample consisting of twenty individuals, both sex, with nonspecific low back pain. Regarding the characterization of the sample, mean height of $1.6895 \pm 0.07547$ was obtained, an average weight of $70.25 \pm 14.97674$, a mean BMI of $24.53 \pm 4.58$ and a mean age of $20.78 \pm 2.65$.

The inclusion criterion was the existence of nonspecific low back pain for a minimum period of three months, with no recent or past diagnosis of low back pain, which was not performing any other type of treatment and was willing to participate in the evaluations. Exclusion criteria were contraindications for the use of any type of electrostimulation and non-attendance on one of the days of data collection. All participants were previously advised about the objectives and the procedures carried out during the study, and signed the Free and Informed Consent Form, previously approved by the institution's Research Ethics Committee (CEP).

Each volunteer was submitted to three sessions of electrostimulation, once a week, with a previous draw in the form of application of the electrostimulation, in a total of three groups of electrostimulation:

i. Conventional TENS Group: frequency of $100 \mathrm{~Hz}$, pulse duration $200 \mu \mathrm{s}$, with comfortable amplitude according to the sensitive threshold of the volunteer, without motor stimulation, during twenty minutes.

ii. Burst TENS Group: frequency modulated at $2 \mathrm{~Hz}$, pulse duration of $200 \mu$ s, with enough amplitude to generate a rhythmic muscle contraction sensation associated with paresthesia for twenty minutes.

iii. VIF TENS Group: variable frequency between 2 to $217 \mathrm{~Hz}$, pulse duration of 50 to $500 \mu$ s and high amplitude, which could cause non-rhythmic fasciculations for twenty minutes.

The device used was Neurodyn II of the Ibramed brand, with the volunteers positioned in a ventral decubitus, electrodes (rubbersilicone $2 \times 4 \mathrm{~cm}$ ) were arranged bilaterally and longitudinally in the lumbar region where the individuals reported pain. A water-soluble gel was applied, aiming to reduce the resistance to current and the electrodes were fixed with adhesive tape in the lumbar region.

After the desired intensity was reached, the time was evaluated, with the purpose of establishing the time of each accommodation. It was explained to the participants that whenever felt a reduction in current intensity should advise the therapist. When the individual reported the accommodation the time was recorded and then the intensity increased to the previous stimulus level during the twenty minutes of the session were noted: the time in seconds of first accommodation and the number of accommodations that occurred during the twenty minutes of therapy. At the end of the therapy the volunteer was asked which current presented the most pleasant.

To verify the normality of the data, the Shapiro-Wilk test was used, since the data presented a non-parametric distribution, the data were presented in median and ranking positions, and compared with the Friedman test, Spearman's correlation was used for the intensity of the current with the accommodation threshold; with respect to the most pleasant current the $\chi 2$ test was used. In all cases, the accepted level of significance was $5 \%$.

\section{Results}

To compare the initial and mean intensity, there was a significant difference between Burst and VIF, with higher values for the first (Table 1).

Table I Initial amplitude for the different groups

\begin{tabular}{lllll}
\hline & Conventional & Burst & VIF & p-value \\
\hline Median & 22 & 27 & 25 & \\
Sum of ranks & 36.5 & $49.5^{*}$ & $34 *$ & 0.0314 \\
\hline
\end{tabular}

*Significant difference between groups

Table 2 Accommodation threshold for the different groups, in seconds (s)

\begin{tabular}{lllll}
\hline & Conventional & Burst & VIF & p-value \\
\hline Median & 144.5 & 267 & 353.5 & \multirow{2}{*}{0.0156} \\
Sum of ranks & $29.5 *$ & $46^{*}$ & 44.5 & \\
\hline
\end{tabular}

*Significant difference between groups

Table 3 Total accommodations

\begin{tabular}{lllll}
\hline & Conventional & Burst & VIF & p-value \\
\hline Median & 5.5 & 4 & 3 & \\
Sum of ranks & $52 *$ & $4 I$ & $27^{*}$ & 0.0004 \\
\hline
\end{tabular}

*Significant difference between groups

For the first accommodation threshold, there was a significant difference between Conventional and Burst, but for the average accommodation there were no differences between groups (Table 2).

For the total of accommodations, during the 20 minutes of therapy, there was a significant difference when comparing the conventional and FIV groups (Table 3).

For the pleasantnessthere were no differences in the three forms of electrostimulation $(\mathrm{p}=0.8187)$. This fact also occurred in the correlation between intensity and accommodation threshold (Conventional $-\mathrm{p}=0.0862$, Burst $-\mathrm{p}=0.8648$, FIV $-\mathrm{p}=0.4863$ )

\section{Discussion}

The main goals in the treatment of chronic low back pain are to relieve pain, increase mobility, improve the quality of life and the physical functions of patients. A widely used method in the physiotherapeutic treatment is TENS, which in several studies has been shown to be effective in chronic pain conditions from different sources. ${ }^{5-8,11-13,16,20}$

In this sense, neural accommodation, defined as the decrease in the perception of the stimulus, is described as an inconvenient factor in the application of TENS. ${ }^{15}$ When the current is applied, the intensity is increased gradually until the individual reports a sensation of intense paresthesia, as soon as this sensation is reduced, that is to say the accommodation occurs, it is indicated that the amplitude of the current is increased so that a constant stimulus is maintained, even for its analgesic effects to be more effective. ${ }^{21,22}$

In this context, there is concern that the phenomenon of accommodation is avoided or at least mitigated, as in the interferential current that has variations in the frequency 
modulated by the amplitude, known as $\Delta \mathrm{F}$, and different forms for its delivery, nevertheless there is still accommodation of the chain. ${ }^{14,23}$ For TENS, there is also electronic instrumentation that seeks to avoid accommodation, with variations in frequency and duration of the phase, called FIP, even being a form that had proven to be more effective than Burst in patients with cancer pain. ${ }^{20}$ As for equipment that does not have such a resource, such as low-frequency transcutaneous electrical nerve stimulation (TENS), it is important in order to increase the intensity of the current to avoid it. ${ }^{22}$

In the present study it can be observed that the intensity variations presented differences only between Burst and VIF, a fact that was already expected, since for the first form the stimulus occurred until muscle contraction was observed, which usually requires higher intensities, however, unexpectedly the intensity was not different for the conventional form. Although there was no difference in intensity, there was a difference between the conventional form and Burst in relation to the accommodation threshold, and the conventional one presented a shorter time until accommodation occurred, without differences between them and the VIF form. The limitation of this study is considered to be the fact that only the first accommodation threshold was evaluated, suggesting that future studies can evaluate the thresholds of all the phenomena that occurred.

Regarding the number of accommodations, there was a significant difference between FIV and the conventional form, indicating that frequency variations and phase durations were effective in decreasing the total accommodation; being that there were no differences with respect to the Burst form, which can be interpreted by the significant difference in intensity of the current used, although the correlations did not present significant differences.

Perceptions of stimulation are quite variable at each level of intensity, and are related to a number of conditions, including skin characteristics such as impedance, temperature, hydration and neural characteristics, such as sensory and nociceptive thresholds, and even aging. ${ }^{24,25}$ Thus, the choice of a current as more pleasant involves several factors, including a possible placebo effect, ${ }^{7}$ and the population studied there was no current higher than the others, in relation to being considered more pleasant. Already Silva et al.comparing 7, 100 and $255 \mathrm{~Hz}$ of TENS in healthy subjects, observed that $7 \mathrm{~Hz}$ was the frequency with the highest number of volunteers indicating as the most pleasant.

\section{Conclusion}

VIF TENS form showed a stimulation that accommodated less, did not require intensities higher than the conventional form, but the time required for the accommodation to occur was not superior to the others. There was no more preferred form of stimulation of the volunteers.

\section{Acknowledgments}

None

\section{Conflicts of interest}

The authors declare that there is no conflicts of interest.

\section{References}

1. Graup S, Bergmann ML, Bergmann GG. Prevalence of nonspecific lumbar pain and associated factors among adolescents in Uruguaiana, state of Rio Grande do Sul. Rev Bras Ortop. 2014;49(6):661-667.

2. Shultz S, Averell K, Eickelman A, et al. Diagnostic accuracy of selfreport and subjective history in the diagnosis of low back pain with nonspecific lower extremity symptoms: A systematic review. Man Ther. 2015;20(1):18-27.

3. Oliveira CB, Maher CG, Pinto RZ, et al. Clinical practice guidelines for the management of non-specific low back pain in primary care: an updated overview. Eur Spine J. 2018;27(11):2791-2803.

4. Petersen $\mathrm{T}$, Olsen S, Laslett M, et al. Inter-tester reliability of a new diagnostic classification system for patients with non-specific low back pain. Aust J Physiother. 2004;50(2):85-94

5. Van Middelkoop M, Rubinstein SM, Kuijpers T, et al. A systematic review on the effectiveness of physical and rehabilitation interventions for chronic non-specific low back pain. Eur Spine J. 2011;20(1):19-39.

6. Itoh $\mathrm{K}$, Itoh S, Katsumi Y, et al. A pilot study on using acupuncture and transcutaneous electrical nerve stimulation to treat chronic non-specific low back pain. Complement Ther Clin Pract. 2009;15(1):22-25.

7. Coutaux A. Non-pharmacological treatments for pain relief: TENS and acupuncture. Jt Bone Spine. 2017;84(6):657-661.

8. Vance CGT, Dailey DL, Rakel BA, et al. Using TENS for pain control: the state of the evidence. Pain Manag. 2014;4(3):197-209.

9. Schulz AP, Chang Chao B, Gazola F, et al. Ação da estimulação elétrica nervosa transcutânea sobre o limiar de dor induzido por pressão/ Transcutaneous electrical nerve stimulation action on pressure-induced pain threshold. Rev Dor. 2011;12(3):231-234

10. Santos CMF, Francischi JN, Lima-Paiva P, et al. Effect of transcutaneous electrical stimulation on nociception and edema induced by peripheral serotonin. Int J Neurosci. 2013;123(7):507-515.

11. Chen CC, Johnson MI. An investigation into the hypoalgesic effects of high- and low-frequency Transcutaneous Electrical Nerve Stimulation (TENS) on experimentally-induced blunt pressure pain in healthy human participants. J Pain. 2010;11(1):53-61.

12. Stein C, Eibel B, Sbruzzi G, et al. Electrical stimulation and electromagnetic field use in patients with diabetic neuropathy: systematic review and metaanalysis. Brazilian J Phys Ther. 2013;17(2):93-104.

13. Simpson PM, Fouche PF, Thomas RE, et al. Transcutaneous electrical nerve stimulation for relieving acute pain in the prehospital setting: A systematic review and meta-analysis of randomized-controlled trials. Eur J Emerg Med. 2014;21(1):10-17.

14. Pivetta KM, Bertolini GRF. $\triangle \mathrm{F}$ efects on the interferential current accommodation in healthy subjects. Rev Bras Med do Esporte. 2012;18(5):330-332

15. Krueger-Beck E, Nogueira-Neto GN, Neves EB, et al. Action potential: from excitation to neural adaptation. Fisioter em Mov. 2011;24(3):535547.

16. Wu LC, Weng PW, Chen $\mathrm{CH}$, et al. Literature review and meta-analysis of transcutaneous electrical nerve stimulation in treating chronic back pain. Reg Anesth Pain Med. 2018;43(4):425-433.

17. Gomes AO, Silvestre AC, Silva CF,et al. Influence of different frequencies of transcutaneous electrical nerve stimulation on the threshold and pain intensity in young subjects. Einstein (Sao Paulo). 2014;12(3):318-322. 
18. Silva DO, Ferreira AS, Gonçalves AV, et al. Efeitos de diferentes frequências da estimulação elétrica nervosa transcutănea em relação à acomodação e à agradabilidade. Sci Med (Porto Alegre). 2014;24(3):264-268.

19. Hughes N, Bennett MI, Johnson MI. An investigation into the magnitude of the current window and perception of transcutaneous electrical nerve stimulation (TENS) sensation at various frequencies and body sites in healthy human participants. Clin J Pain. 2013;29(2):146-153.

20. Schleder JC, Verner FA, Mauda L, et al. The transcutaneous electrica nerve stimulation of variable frequency intensity has a longer-lasting analgesic action than the burst transcutaneous electrical nerve stimulation in cancer pain. Rev Dor. 2017;18(4):316-320.

21. Poitras S, Brosseau L. Evidence-informed management of chronic low back pain with transcutaneous electrical nerve stimulation, interferential current, electrical muscle stimulation, ultrasound, and thermotherapy. Spine J. 2008;8(1):226-233.
22. Pantaleão MA, Laurino MF, Gallego NLG, et al. Adjusting pulse amplitude during Transcutaneous Electrical Nerve Stimulation (TENS) application produces greater hypoalgesia. J Pain. 2011;12(5):581-590.

23. Guerra TEC, Bertolini GRF. Effects of $\Delta \mathrm{F}$ delivery ramp variation on the accommodation of the interferential current in healthy women. Rev Dor. 2012;13(1):25-29.

24. Boada MD, Eisenach JC, Ririe DG. Mechanical sensibility of nociceptive and non-nociceptive fast-conducting afferents is modulated by skin temperature. J Neurophysiol. 2016;115(1):546-553.

25. Vance CGT, Chimenti RL, Dailey DL, et al. Development of a method to maximize the transcutaneous electrical nerve stimulation intensity in women with fibromyalgia. J Pain Res. 2018;11:2269-2278. 